Leziak Milena, Niedobylski Sylwiusz, Żak Klaudia, Piwoński Michał, Krasowska Danuta, Skórzyńska-Dziduszko Katarzyna. Oxytocin and postpartum depression - a possible treatment for depressed mothers. Journal of Education, Health and Sport. 2020;10(7):161-172. eISSN 2391-8306. DOI http://dx.doi.org/10.12775/JEHS.2020.10.07.016

https://apcz.umk.pl/czasopisma/index.php/JEHS/article/view/JEHS.2020.10.07.016

https://zenodo.org/record/3953470

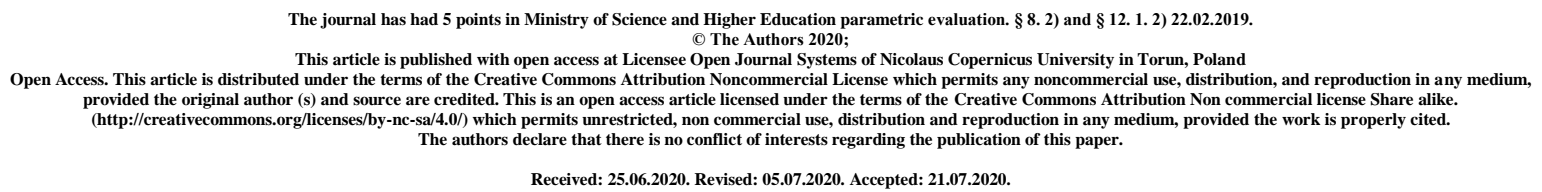

\title{
Oxytocin and postpartum depression - a possible treatment for depressed mothers
}

\author{
Milena Leziak, Sylwiusz Niedobylski, Klaudia Żak, Michał Piwoński, \\ Danuta Krasowska, Katarzyna Skórzyńska-Dziduszko
}

Milena Leziak, e-mail: milena.leziak@wp.pl, ORCID: https://orcid.org/0000-0001-99115534 (1),

Sylwiusz Niedobylski, e-mail: sniedobylski@gmail.com, ORCID : https://orcid.org/0000$\underline{0001-7266-623 X}(1)$,

Klaudia Żak, e-mail: zakklaudia3@gmail.com, ORCID : https://orcid.org/0000-0003-2421$\underline{2553}(1)$,

Michał Piwoński, e-mail: michalpiwonski2@gmail.com, ORCID: https://orcid.org/00000001-6510-8993 (1),

Danuta Krasowska, e-mail: dana.krasowska@gmail.com, ORCID: https://orcid.org/00000002-3015-1120 (2),

Katarzyna Skórzyńska-Dziduszko, e-mail: katarzynaskorzynskadziduszko@umlub.pl, ORCID: http://orcid.org/0000-0002-8718-1187 (3)

Affiliation: 1 - Students' Scientific Group at the Chair and Department of Human Physiology, Medical University of Lublin

2 - Students' Scientific Group at the Chair and Department of Dermatology, Venereology and Pediatric Dermatology, Medical University of Lublin

3 - Department of Human Physiology, Medical University of Lublin 


\begin{abstract}
Introduction and purpose: Postpartum depression (PPD) is one of the most common conditions in childbearing. It is estimated that 10-15\% of mothers develop PPD. Oxytocin has recently been implicated in the pathophysiology of several neuropsychiatric disorders including PPD. The aim of this study is to, on the base of recent studies, investigate whether oxytocin has an impact on PPD and if it can be used as a way of diagnostic indicator or treatment.
\end{abstract}

Brief description of the state of knowledge: Studies indicate that there is a relationship between oxytocin and PPD. Depression and anxiety after delivery were observed more frequently among women with lower oxytocin plasma levels. Basing on the oxytocin plasma levels in the third semester of pregnancy, the risk of PPD can be predicted. This relationship was observed in women who were breastfeeding as well as in those who ceased. Some studies indicate that depression development may be inhibited thanks to oxytocin. Exogenous oxytocin has been proven to improve prosocial behaviour and reduce negative emotional responses in mother-children relations. It may also increase mother's attention to baby. Some studies have stated that intrapartum oxytocin administration decreases the rate of PPD symptoms but studies on treatment with oxytocin are not unanimous.

Conclusions: Oxytocin plays an undeniable role in pathophysiology of postpartum depression and it may be regarded as a diagnostic indicator and therapeutic target. However, more studies are needed to clarify this intricate relationship.

Key words: oxytocin; postpartum depression; pregnancy; maternal behavior; depression

\title{
Introduction and objective
}

For the majority of women, having a baby is a time full of joy, happiness and a feeling of maternal fulfillment. Pregnancy and the period after child delivery may be an especially vulnerable time for mothers. During pregnancy women often experience intense emotional and biological changes, which together with risk factors, may lead to development of different mental disorders. Postpartum depression (PPD) is one of the most common and disabling conditions of childbearing, but it is treatable. It is estimated that $10-15 \%$ of women develop PPD [1].

PPD is characterized by depressed mood, feelings of guilt and worthlessness, anhedonia, reduced energy, crying for "no reason", sleep and appetite disturbances, low self-esteem, cognitive dysfunction and even suicidal thoughts [2]. These symptoms cause a functioning revolution in mother's behavior, diminishing a woman's quality of life, influences her social relationships and especially causes an impairment in the mother-child bond. In consequence, such distress may have a substantially negative impact on the child's proper cognitive, social, behavioral, motor and emotional development [3]. According to the American Psychiatric Association's Diagnostic and Statistical Manual of Mental Disorders, 5th edition PPD can be diagnosed, if the onset of symptoms occur during pregnancy or in the 4 weeks following childbirth [4]. But, a number of clinicians and researchers assume that the occurrence of PPD varies from 4 weeks to even 12 months after delivery [5].

The pathophysiology of PPD depression is complex and not fully determined. However, there are various risk factors including biological, psychological, socioeconomic, genetic, immune 
and hormonal factors that contribute to the onset of PPD [2]. Major factors include: previous medical or family history of depression or other anxiety disorders; high risk pregnancy (preterm birth, the infant's impaired functioning, stress during pregnancy); low socioeconomic status.

Recently, a lot of attention was paid to oxytocin and its relationship with postpartum depression. This hormone, whose main functions are stimulation of milk production and uterine contraction, also plays an important role in social recognition and bonding [6]. The aim of this study was to review the current literature on the relationship between oxytocin and postpartum depression as well as its therapeutic use in this condition. Evidence gathered here may guide further investigation and clinical recommendations.

This review presents recent studies available in PubMed base, investigating the relationship between oxytocin and postpartum depression (studies from 2016-2020) or treatment of PPD with oxytocin and its results. We included studies on humans as well as on animals.

\section{Results}

\section{Endogenous oxytocine}

The aim of the first presented study was to analyze if OXT plasma levels has an association with affective symptoms in women during the third trimester of pregnancy. 13 women with an affective disorder (treated during examination) were compared to 9 women with preeclampsia and 12 women without disorders, overall 25 patients took part in this study. Two groups were distinguished, accordingly to oxytocin levels: one of them with the OXT smaller than $45 \mathrm{pg} / \mathrm{mL}$ and the second one with $45 \mathrm{pg} / \mathrm{mL}$ or more. Independently of other disorders, patients with OXT plasma levels smaller than $45 \mathrm{pg} / \mathrm{mL}$ featured worse on the Edinburgh Postnatal Depression Scale (EPDS) and the State/Trait Anxiety Inventory Form Y (STAI-Y) state total scores in comparison to women with OXT plasma $45 \mathrm{pg} / \mathrm{mL}$ or more $(\mathrm{p}=0,01)$. Except of this, no statistically significant difference was noticed between 3 researched groups. On this account the most important conclusion was formed: depression and anxiety are more frequent among women with lower OXT plasma levels, regardless of the diagnosis of preeclampsia and affective disorder [7].

Authors of another study drew blood samples of 74 pregnant women in the third semester of pregnancy (between $30^{\text {th }}$ and $34^{\text {th }}$ week of gestation). Their purpose was to check if prenatal OXT plasma level is a potential predictor of the risk of PPD. To assess depressive symptoms the EPDS scale was used and women were divided into two groups -14 participants with 10 or more points in the EPDS scale were at risk of PPD development (rPPD group) and the rest -59 participants in nPPD group. Plasma OXT level significantly predicted PPD symptoms $(p<0,05)$ - OXT levels were lower in the rPPD group. In 83,6\% of cases, the OXT plasma level confirmed a correct qualifying into a given group [8].

Jobst et al. evaluated 100 women in the $35^{\text {th }}$ and $38^{\text {th }}$ week of pregnancy and three postpartum women: 2 days, 7 weeks and 6 months after delivery. "Depressed" group was qualified using the Montgomery-Asberg Depression Rating Scale on which patients got 10 or more points. In all the participants, the levels of plasma OXT were higher 6 months postpartum than in the $35^{\text {th }}$ week of gestation. But the biggest difference was between the "depressed" group, in which 2 days after delivery OXT plasma levels decreased compared to 
the $38^{\text {th }}$ week of gestation and the "non-depressed" group in which OXT plasma levels were higher 2 days after delivery in the comparison to the postpartum period [9].

66 healthy pregnant women in the third trimester and at 6 weeks postpartum were evaluated with the aim to verify the interdependence between the OXT level and depressive symptom severity. The oxytocin level was examined during the pregnancy and after the delivery, depressive symptoms were measured using EPDS. 13 patients had a previous major depressive episode and 53 have not such episodes. In the third trimester depressive symptoms were not associated with the oxytocin level $(\mathrm{p}=0,19)$. The oxytocin level (the report from the third trimester) was not useful to predict PPD symptom severity in women without major depressive disorder (MDD) $(p=0,219)$, nonetheless in the case of women with MDD it was helpful $(\mathrm{p}=0,003)[10]$.

Some associations between Postpartum Depression, Breastfeeding and Oxytocin Level among Latina Mothers were described by Sandraluz Lara-Cinisomo et al. 34 Latina Mothers were observed from their third trimester of pregnancy until 8 weeks postpartum. 2 scales were used: EPDS and STAI. 8 women $(n=8)$ were depressed at the beginning of the research and in the 8-week laboratory visit 6 women had more than 10 points in the EPDS scale. It has been observed that women who were depressed at 8 weeks postpartum and stopped breastfeeding had lower OXT levels than women who were not depressed and stopped breastfeeding $(\mathrm{p}=0,044)$. In addition, women who stopped breastfeeding before 8 weeks postpartum had higher EPDS subscale scores $(\mathrm{p}=0,002)$ at this stage ( 8 weeks postpartum) [11].

Stuebe et al. recruited 52 women in their third trimester of pregnancy, who were examined psychiatrically with the depression and anxiety disorder modules of the Structured Clinical Interview Non-Patient version (SCID-NP) usage. The blood samples were also drawn from the participants for OXT levels assessment. Mothers completed the Edinburgh Postnatal Depression Scale (EPDS) and the Spielberger State and Trait Anxiety Inventories (STAI). Lower OXT levels during breastfeeding were stated to be inversely correlated with maternal EPDS and STAI-State and STAI-Trait anxiety scores among 39 women who breastfed at 8 weeks postpartum, whereas lower oxytocin levels were denoted in case of higher anxiety and depression scores during feeding [12].

The authors of another work (Cox et al.) took into account the results of the same group of women as Stuebe et al., who were subjected to a standardized stressor, the Trier Social Stress Test (TSST) at 8 weeks postpartum - 39 of them were breastfeeding during the period when TSST was performed. Mood symptoms were assessed using the EDPS scale and Spielberger. Mothers' OXT blood levels were quantified as well. Symptomatic breastfeeding women tended to manifest lower OXT levels during breastfeeding with higher cortisol levels both during breastfeeding and the TSST. There was also a positive correlation between OXT and cortisol levels during TSST (instead of the expected negative correlation, which was found among asymptomatic women). Thus, among symptomatic women, OXT may increase cortisol-dependent response to stress, while in asymptomatic women - may decrease. The results of the work suggest the possible role of dysregulation of OXT and hypothalamuspituitary-adrenal glands axis in PPD pathogenesis in breastfeeding women [13].

Apter Levy et al., in their work recruited a community cohort women representing high or low depression scores two days after childbirth. As it was stated, among depressed mothers it was more likely than among the nondepressed ones to have the GG genotype for 
OXT receptor gene rs2254298. What's more, in mothers with the GG genotype lower salivary OXT levels were assessed than in those with the AG/AA genotypes. Genetic and peripheral biomarkers of the oxytocin system seem to imply poorer child social outcomes in chronically depressed women, which may suggest the legitimacy for oxytocin-based interventions development in search for diminishing the PPD risks [14].

Zelkowitz et al. took into account a community sample of 287 women with plasma OXT measurement, maternal symptoms of depression using The Edinburgh Postnatal Depression Scale (EPDS) and psychosocial stress with The Antenatal Risk Questionnaire (ANRQ) usage. It turns out that there is no association between OXT plasma levels and a mother's depressive symptoms [15]. However, in case of conclusions of Garfield et al., drawn based on the results of 57 women who were asked to complete surveys (the Center for Epidemiologic Studies Depression Scale (CESD) for depression assessment and the StateTrait Anxiety Inventory (STAI) for anxiety assessment) and who had blood drawn for OXT level assessment, depressive symptoms tend to be higher in women with low OXT levels in comparison to women with high levels of this neurohormone [16]. Nevertheless, according to Zelkowitz et al., in women with high psychosocial stress levels, fewer depressive symptoms were associated with OXT plasma levels higher than in case of the women with lower OXT plasma levels. These results may be suggesting that major depression development may be inhibited thanks to OXT.

The aim of the work of Whitley et al. was to show the relationship between anxiety, oxytocin, breastfeeding and PPD. A group of 222 women were asked to complete the Beck Depression Inventory II (the BDI-II) and Spielberger State-Trait Anxiety Inventory-state subscale (STAI-S) provide venous blood samples drawn during breastfeeding period to assess OXT levels and take part in infant feeding sessions at 2 and 6 months postpartum. According to the results, there were no significant differences in OXT levels across a breastfeeding sessions between depressed or anxious women nor asymptomatic ones. No differences in oxytocin fluctuations were demonstrated during breastfeeding in symptomatic group but possible differences in case of antidepressant use [17].

\section{Exogenous oxytocine}

The first animal study on OXT treatment was conducted by Cort A. Pedersen and Arthur J. Prange Jr. and published in 1979 - virgin female rats were administered with OXT through lateral ventricular cannulae - within 2 hours $42 \%$ of them started to display maternal behaviour towards foster pups and - allowed to stay with them for next 10 days - continued to behave the same way [18].

The first time that antidepressive effect of OXT was proven was in 1987 study which based on behavioural despair test in mice- the peptide was administered in one group of animals and its efficacy was compared with that of imipramine. In the helplessness test OXT significantly reduced mean immobility time which is the indicator of negative mood, with the effect being even more pronounced than that of imipramine [19]. One of many examples of such results is 2018 study on depressive behaviours in the PPD rat model - local administration of 20ng OXT into paraventricular nucleus decreased severity of all features assessed in immobility test and lowered mean plasma corticosterone in depressed specimens [20]. 2017 study of Abdul Shukkoor et al. showed interesting method of dealing with PPD - specimens of PPD rat model was administered with lipid extract of freshwater fish Channa striatus which caused 
alleviation of depressive symptoms in behavioural despair test, and one of proposed mechanisms for that effect is an increase in plasma OXT caused by the extract [21].

Exogenous OXT has been proven to improve prosocial behaviour in humans as well - Ditzen et al. designed a double-blind placebo-controlled study which involved intranasal administration of OXT in 47 heterosexual couples and compared their communication skills (which involved nonverbal signs as well) and salivary cortisol during standard instructed couple conflict discussion with placebo group - the OXT group displayed significantly more positive communication patterns and lower mean salivary cortisol level after the discussion [22]. In different study patients after intranasal OXT administration had higher skill of inferring mental state of other people by interpreting subtle social cues in Reading the Mind in the Eyes Test compared with placebo [23]. A 2008 study shows abolishment of negative evaluation of aversively conditioned faces in response to treatment with OXT [24]. Similar result can be seen in 2018 study on mothers and their response to emotional infant and adult faces which was measured through cortical event-related potentials recordings. Mothers on OXT displayed more pronounced responses to faces of both infants and adults without significant differences [25]. The existence of said paper and others are the evidence that influence of exogenous OXT on mother-children relations is the point of interest of many researchers - a group of them - Riem et al. - tested amygdala, insula and inferior frontal gyrus responses to crying infant sounds in mothers after intranasal OXT administration. Magnetic resonance imaging showed reduced activation in aversion-related regions, and upgrade in neural circuitry for empathy [26]. In 2012 study mothers after intranasal OXT used lower handgrip force in response to infant crying sound compared to placebo - the effect was seen only in mothers with positive personal early parenting experiences [27]. In yet another study Riem et al. tested the influence of synthetic OXT on the response to infant laughter brains of mothers involved in the study displayed upgraded functional connectivity between the amygdala and emotional-response-related regions which may enhance the incentive salience of infant laughter and reduce negative emotional responses [28]. Holtfrerich et al. shed light on even different approach to OXT action - knowing that higher endogenous testosterone levels reduce mothers attention to infants the researchers tested the response of mothers with different serum levels of that hormone to baby schema after OXT administration. It turned out that OXT increased women attention to baby schema among adult distractors and the effect was notably pronounced in mothers with higher testosterone levels (compared to results of the same women before OXT administration), which may indicate that OXT acted positively by alleviation of negative testosterone effects [29].

Exogenous OXT turned out to be effective in various psychological/psychiatric conditions as well, examples of which include its therapeutic effect on positive, negative and cognitive domains of schizophrenia [30], improvement of social interaction deficits in autistic children [31], enhancement of the experience of attachment security [32] or attenuation of stress reactivity in borderline personality disorder [33].

It's efficacy was tested in depression [34] as well, involving PPD. Takács et al. conducted a prospective observational study on the effects of intrapartum synthetic OXT on mothers postnatal mood. 260 patients were screened for symptoms of depression while pregnant and 2 times postpartum. After taking intrapartum OXT administration into account the women that received said synthetic peptide displayed significantly lower rate of PPD symptoms (hazard 
ratio: 0.65$)$. OXT didn't affect maternal mood immediately, it didn't had any effect on maternity blues as well [35]. Lorenz et al. did an analysis of functional imaging of brain regions important to emotion and reward processing in search for activation in response to sexual, and infant images in PPD mothers. 23 participants were divided to receive intranasal OXT or placebo. Among OXT patients all the changes were similar to those of women on antidepressants - one of which was significantly higher amygdala activation in response to sexual images compared to placebo group with less spectacular changes in response to other stimuli [36]. In contrast there are studies on the topic which results didn't meet the researchers expectations. In 2017 a population-based study was published the authors of which evaluated the relative risk of PPD and anxiety disorders in 9684 patients exposed on OXT peripartum and 37048 unexposed individuals. Exposure to exogenous OXT turned out to increase the risk of depressive or anxiety disorders postpartum by $36 \%$ and $32 \%$ in patients with and without depressive or anxiety disorders history, respectively within the first year after labor [37]. Beth L. Mah et al. wanted to check the influence of treatment with intranasal OXT on mothers with a diagnosis of postnatal depression (PND): their mood and expressed emotions. It was a randomized, double-blind, placebo controlled, within-subject study in which 25 mothers took part (range 19-38 with mean age 28,24 years). Intranasal OXT was administered alternately with placebo. Postnatal depression was measured by Edinburgh Post Natal Depression Scale (EPNDS), for evaluation of the current mood the Self-Assessment Manikin was used, Five Minute Speech Sample was applied to expressed emotion assessment and the last one: the Controlled Oral Word Association to test mothers' ability to focus on one task only. The mothers in the OXT group described their infants as more difficult, but the quality of the relationship with babies was more positive $(p=0,036)$. The presence of PND was independent of the intranasal OXT admission $(\mathrm{p}=0.85)$, moreover they were sadder than the mothers in the placebo group $(\mathrm{p}=0,01)$. The results of this study didn't meet the expectations of the authors - they assumed that in the OXT group not only the relationship, but also the description of their infant would be more positive. However, in most cases, mothers described their baby in a negative way, and then added/explained how close they and their children were [38]. One of the explanations of negative exogenous intrapartum OXT influence on mothers postnatal mood may be decreased endogenous OXT production in response to exogenous OXT administration [39].

\section{Mechanisms}

Oxytocin reduces stress and increases the occurrence of pro-social cues thereby facilitating trust, bonding, social support seeking, empathy, attachment and social cognition [40]. Oxytocin has recently been implicated in the pathophysiology of several neuropsychiatric disorders including postpartum depression, but the relationship is not well understood.

What is known is that oxytocin suppresses hypothalamic-pituitary-adrenal (HPA) axis over activity by reducing excess cortisol. Abnormalities in oxytocin signalling dysregulate the HPA axis. Jolley et al. studied postpartum women who underwent a physical stressor. Postpartum women with depression showed no relationship between their ACTH and cortisol levels, with higher ACTH and lower cortisol levels when compared with the nondepressed group, in which the HPA axis was regulated [41]. This may be one of the possible ways of oxytocin influencing postpartum depression occurrence. 
Another possibility was proposed in Shahrokh's et al. study. It demonstrated a direct effect of oxytocin on dopamine release within the mesocorticolimbic dopamine system. Oxytocin neurons in the medial preoptic area were shown to regulate dopamine function in the ventral tegmental area, thereby determining the rewarding properties. It is possible that with improper oxytocin signaling, reward properties are altered, which could lead to changes in maternal behaviour and mood [42][13].

Not only oxytocin level but also its receptors may play an important role in pathogenesis of postpartum depression. It was studied that women with persistent perinatal depression had significantly higher methylation of oxytocin receptor gene than women without perinatal depression [40]. This association was also confirmed in another study. Similar patterns of methylation density in oxytocin receptor gene were observed in women with perinatal depression and with insecure attachment style, which indicates association between those two characteristics [43]. Moreover, it was proposed that infants' oxytocin receptor genotype might also alter the behaviour of mothers who exhibit depressive characteristics [44].

Oxytocin seems to interact also with serotonergic system. Within the raphe nucleus, a subpopulation of serotonergic neurons expresses receptors for oxytocin. Thus, oxytocin based therapy might be an additional option for depressed individuals [45].

\section{Conclusions}

Results of the presented studies indicate that there is an undeniable relationship between oxytocin and PPD. Depression and anxiety are more frequent among women with lower OXT plasma level. This hormone may be regarded as a diagnostic and therapeutic indicator in peripartum and postpartum depression in women, and, what is more, it can be considered as a treatment for PPD. Especially considering that some of presented studies indicate that depression development may be inhibited thanks to OXT. More studies and trials are necessary to confirm and clarify this intricate relationship, but it is to be hoped that oxytocin can help at least some of depressed mothers.

\section{Bibliography}

[1] Bennett HA, Einarson A, Taddio A, Koren G, Einarson TR. Prevalence of depression during pregnancy: Systematic review. Obstet Gynecol 2004;103:698-709. https://doi.org/10.1097/01.AOG.0000116689.75396.5f.

[2] Stewart DE, Vigod SN. Postpartum Depression: Pathophysiology, Treatment, and Emerging Therapeutics. Annu Rev Med 2019;70:183-96. https://doi.org/10.1146/annurev-med-041217-011106. 
[3] Malus A, Szyluk J, Galinska-Skok B, Konarzewska B. Incidence of postpartum depression and couple relationship quality. Psychiatr Pol 2016;50:1135-46. https://doi.org/10.12740/PP/61569.

[4] American Psychiatric Association. Diagnostic and Statistical Manual of Mental Disorders. American Psychiatric Association; 2013. https://doi.org/10.1176/appi.books.9780890425596.

[5] Stewart DE, Vigod S. Postpartum Depression. N Engl J Med 2016;375:2177-86. https://doi.org/10.1056/NEJMcp1607649.

[6] Kendrick KM, Guastella AJ, Becker B. Overview of human oxytocin research. Curr. Top. Behav. Neurosci., vol. 35, Springer Verlag; 2018, p. 321-48. https://doi.org/10.1007/7854_2017_19.

[7] Serati M, Grassi S, Redaelli M, Pergoli L, Cantone L, La Vecchia A, et al. Is There an Association Between Oxytocin Levels in Plasma and Pregnant Women's Mental $\begin{array}{llllll}\text { Health? } & \mathrm{J} & \text { Am } & \text { Psychiatr }\end{array}$ https://doi.org/10.1177/1078390319890400.

[8] Skrundz M, Bolten M, Nast I, Hellhammer DH, Meinlschmidt G. Plasma oxytocin concentration during pregnancy is associated with development of postpartum depression. Neuropsychopharmacology 2011;36:1886-93. https://doi.org/10.1038/npp.2011.74.

[9] Jobst A, Krause D, Maiwald C, Härtl K, Myint AM, Kästner R, et al. Oxytocin course over pregnancy and postpartum period and the association with postpartum depressive symptoms. Arch Womens Ment Health 2016;19:571-9. https://doi.org/10.1007/s00737-016-0644-2.

[10] Massey SH, Schuette SA, Pournajafi-Nazarloo H, Wisner KL, Carter CS. Interaction of oxytocin level and past depression may predict postpartum depressive symptom severity. Arch Womens Ment Health 2016;19:799-808. https://doi.org/10.1007/s00737-016-0616-6.

[11] Lara-Cinisomo S, McKenney K, Di Florio A, Meltzer-Brody S. Associations Between Postpartum Depression, Breastfeeding, and Oxytocin Levels in Latina Mothers. Breastfeed Med 2017;12:436-42. https://doi.org/10.1089/bfm.2016.0213.

[12] Stuebe AM, Grewen K, Meltzer-Brody S. Association between maternal mood and oxytocin response to breastfeeding. J Women's Heal 2013;22:352-61. https://doi.org/10.1089/jwh.2012.3768.

[13] Cox EQ, Stuebe A, Pearson B, Grewen K, Rubinow D, Meltzer-Brody S. Oxytocin and HPA stress axis reactivity in postpartum women. Psychoneuroendocrinology 2015;55:164-72. https://doi.org/10.1016/j.psyneuen.2015.02.009.

[14] Apter-Levy Y, Feldman M, Vakart A, Ebstein RP, Feldman R. Impact of maternal depression across the first 6 years of life on the child's mental health, social engagement, and empathy: The moderating role of oxytocin. Am J Psychiatry 2013;170:1161-8. https://doi.org/10.1176/appi.ajp.2013.12121597.

[15] Zelkowitz P, Gold I, Feeley N, Hayton B, Carter CS, Tulandi T, et al. Psychosocial stress moderates the relationships between oxytocin, perinatal depression, and maternal behavior. Horm Behav 2014;66:351-60. https://doi.org/10.1016/j.yhbeh.2014.06.014.

[16] Garfield L, Giurgescu C, Carter CS, Holditch-Davis D, McFarlin BL, Schwertz D, et 
al. Depressive symptoms in the second trimester relate to low oxytocin levels in African-American women: a pilot study. Arch Womens Ment Health 2015;18:123-9. https://doi.org/10.1007/s00737-014-0437-4.

[17] Whitley J, Wouk K, Bauer AE, Grewen K, Gottfredson NC, Meltzer-Brody S, et al. Oxytocin during breastfeeding and maternal mood symptoms. Psychoneuroendocrinology 2020;113:104581. https://doi.org/10.1016/j.psyneuen.2019.104581.

[18] Pedersen CA, Prange AJ. Induction of maternal behavior in virgin rats after intracerebroventricular administration of oxytocin. Proc Natl Acad Sci 1979;76:66615. https://doi.org/10.1073/pnas.76.12.6661.

[19] Arletti R, Bertolini A. Oxytocin acts as an antidepressant in two animal models of depression. Life Sci 1987;41:1725-30. https://doi.org/10.1016/0024-3205(87)90600-X.

[20] Wang T, Shi C, Li X, Zhang P, Liu B, Wang H, et al. Injection of oxytocin into paraventricular nucleus reverses depressive-like behaviors in the postpartum depression rat model. Behav Brain Res 2018;336:236-43. https://doi.org/10.1016/j.bbr.2017.09.012.

[21] Abdul Shukkoor MS, Baharuldin MTH Bin, Mat Jais AM, Mohamad Moklas MA, Fakurazi S, Basir R. Antidepressant-Like Effect of Lipid Extract of Channa striatus in Postpartum Model of Depression in Rats. Evidence-Based Complement Altern Med ECAM 2017;2017:1469209. https://doi.org/10.1155/2017/1469209.

[22] Ditzen B, Schaer M, Gabriel B, Bodenmann G, Ehlert U, Heinrichs M. Intranasal Oxytocin Increases Positive Communication and Reduces Cortisol Levels During Couple Conflict. Biol Psychiatry 2009;65:728-31. https://doi.org/10.1016/j.biopsych.2008.10.011.

[23] Domes G, Heinrichs M, Michel A, Berger C, Herpertz SC. Oxytocin Improves "MindReading" in Humans. Biol Psychiatry 2007;61:731-3. https://doi.org/10.1016/j.biopsych.2006.07.015.

[24] Petrovic P, Kalisch R, Singer T, Dolan RJ. Oxytocin Attenuates Affective Evaluations of Conditioned Faces and Amygdala Activity. J Neurosci 2008;28:6607-15. https://doi.org/10.1523/JNEUROSCI.4572-07.2008.

[25] Peltola MJ, Strathearn L, Puura K. Oxytocin promotes face-sensitive neural responses to infant and adult faces in mothers. Psychoneuroendocrinology 2018;91:261-70. https://doi.org/10.1016/j.psyneuen.2018.02.012.

[26] Riem MME, Bakermans-Kranenburg MJ, Pieper S, Tops M, Boksem MAS, Vermeiren RRJM, et al. Oxytocin modulates amygdala, insula, and inferior frontal gyrus responses to infant crying: a randomized controlled trial. Biol Psychiatry 2011;70:291-7. https://doi.org/10.1016/j.biopsych.2011.02.006.

[27] Bakermans-Kranenburg MJ, van Ijzendoorn MH, Riem MME, Tops M, Alink LRA. Oxytocin decreases handgrip force in reaction to infant crying in females without harsh parenting experiences. Soc Cogn Affect Neurosci 2012;7:951-7. https://doi.org/10.1093/scan/nsr067.

[28] Riem MME, van IJzendoorn MH, Tops M, Boksem MAS, Rombouts SARB, Bakermans-Kranenburg MJ. No laughing matter: intranasal oxytocin administration changes functional brain connectivity during exposure to infant laughter. 
Neuropsychopharmacol Off Publ Am Coll Neuropsychopharmacol 2012;37:1257-66. https://doi.org/10.1038/npp.2011.313.

[29] Holtfrerich SKC, Pfister R, El Gammal AT, Bellon E, Diekhof EK. Endogenous testosterone and exogenous oxytocin influence the response to baby schema in the female brain. Sci Rep 2018;8:7672. https://doi.org/10.1038/s41598-018-26020-4.

[30] Feifel D, Shilling PD, MacDonald K. A Review of Oxytocin's Effects on the Positive, Negative, and Cognitive Domains of Schizophrenia. Biol Psychiatry 2016;79:222-33. https://doi.org/10.1016/j.biopsych.2015.07.025.

[31] Yatawara CJ, Einfeld SL, Hickie IB, Davenport TA, Guastella AJ. The effect of oxytocin nasal spray on social interaction deficits observed in young children with autism: a randomized clinical crossover trial. Mol Psychiatry 2016;21:1225-31. https://doi.org/10.1038/mp.2015.162.

[32] Buchheim A, Heinrichs M, George C, Pokorny D, Koops E, Henningsen P, et al. Oxytocin enhances the experience of attachment security. Psychoneuroendocrinology 2009;34:1417-22. https://doi.org/10.1016/j.psyneuen.2009.04.002.

[33] Simeon D, Bartz J, Hamilton H, Crystal S, Braun A, Ketay S, et al. Oxytocin administration attenuates stress reactivity in borderline personality disorder: A pilot study. Psychoneuroendocrinology 2011;36:1418-21. https://doi.org/10.1016/j.psyneuen.2011.03.013.

[34] Pincus D, Kose S, Arana A, Johnson K, Morgan PS, Borckardt J, et al. Inverse effects of oxytocin on attributing mental activity to others in depressed and healthy subjects: a double-blind placebo controlled FMRI study. Front Psychiatry 2010;1:134. https://doi.org/10.3389/fpsyt.2010.00134.

[35] Takács L, Seidlerová JM, Štěrbová Z, Čepický P, Havlíček J. The effects of intrapartum synthetic oxytocin on maternal postpartum mood: findings from a prospective observational study. Arch Womens Ment Health 2019;22:485-91. https://doi.org/10.1007/s00737-018-0913-3.

[36] Lorenz TK, Cheng H, Heiman JR. Neural correlates of emotion processing comparing antidepressants and exogenous oxytocin in postpartum depressed women: An $\begin{array}{llll}\text { exploratory } & \text { study. } & \text { PLoS } & \text { One }\end{array}$ https://doi.org/10.1371/journal.pone.0217764.

[37] Kroll-Desrosiers AR, Nephew BC, Babb JA, Guilarte-Walker Y, Moore Simas TA, Deligiannidis KM. Association of peripartum synthetic oxytocin administration and depressive and anxiety disorders within the first postpartum year. Depress Anxiety 2017;34:137-46. https://doi.org/10.1002/da.22599.

[38] Mah BL, Van Ijzendoorn MH, Smith R, Bakermans-Kranenburg MJ. Oxytocin in postnatally depressed mothers: its influence on mood and expressed emotion. Prog Neuropsychopharmacol Biol Psychiatry 2013;40:267-72. https://doi.org/10.1016/j.pnpbp.2012.10.005.

[39] Jonas K, Johansson LM, Nissen E, Ejdebäck M, Ransjö-Arvidson AB, Uvnäs-Moberg K. Effects of intrapartum oxytocin administration and epidural analgesia on the concentration of plasma oxytocin and prolactin, in response to suckling during the second day postpartum. Breastfeed Med Off J Acad Breastfeed Med 2009;4:71-82. https://doi.org/10.1089/bfm.2008.0002. 
[40] King L, Robins S, Chen G, Yerko V, Zhou Y, Nagy C, et al. Perinatal depression and DNA methylation of oxytocin-related genes: a study of mothers and their children. Horm Behav 2017;96:84-94. https://doi.org/10.1016/j.yhbeh.2017.09.006.

[41] Jolley SN, Elmore S, Barnard KE, Carr DB. Dysregulation of the hypothalamicpituitary-adrenal axis in postpartum depression. Biol Res Nurs 2007;8:210-22. https://doi.org/10.1177/1099800406294598.

[42] Shahrokh DK, Zhang TY, Diorio J, Gratton A, Meaney MJ. Oxytocin-dopamine interactions mediate variations in maternal behavior in the rat. Endocrinology 2010;151:2276-86. https://doi.org/10.1210/en.2009-1271.

[43] Robakis TK, Zhang S, Rasgon NL, Li T, Wang T, Roth MC, et al. Epigenetic signatures of attachment insecurity and childhood adversity provide evidence for role transition in the pathogenesis of perinatal depression. Transl Psychiatry 2020;10:1-14. https://doi.org/10.1038/s41398-020-0703-3.

[44] Asherin RM, Everhart KD, Stophaeros SL, Vogeli JM, Fowler J, Phiel CJ, et al. Associations between maternal depression and mother and infant oxytocin receptor gene (OXTR_rs53576) polymorphisms. Dev Psychobiol 2019:dev.21938. https://doi.org/10.1002/dev.21938.

[45] Neumann ID, Landgraf R. Balance of brain oxytocin and vasopressin: Implications for anxiety, depression, and social behaviors. Trends Neurosci 2012;35:649-59. https://doi.org/10.1016/j.tins.2012.08.004. 